\title{
Metallic Copper Clusters Decorating Cu Ferrites Revealed by Deep Data Analysis
}

\author{
Philippe Kikongi ${ }^{1}$, Nicolas Dumaresq ${ }^{1}$, Francis B. Lavoie ${ }^{1}$, Ryan Gosselin ${ }^{1}$ and Nadi Braidy ${ }^{1}$ \\ ${ }^{1}$ Chemical and Biotechnological Engineering, Université de Sherbrooke, Sherbrooke (QC) Canada
}

With improved performance of TEMs and spectrometers, the limiting step for better materials characterization is now the ability to translate large datasets into meaningful information. Here, we present a new, semi-supervised method for retrieving spectral components and their respective spatial contribution that is efficient when weak spectral features are scattered in the spectrum-image (SI).

The sample is made of nanoparticles (NPs) produced by injecting an equimolar ratio of $\mathrm{Cu}$ and $\mathrm{Fe}$ nitrates solution in an induction $\mathrm{Ar} / \mathrm{O}_{2}$ plasma reactor. $\mathrm{XRD}$ analyses indicated the presence of $\mathrm{CuFe}_{2} \mathrm{O}_{4}$ tetragonal phase (33\% wt), $\mathrm{Cu}_{0.5} \mathrm{Fe}_{2.5} \mathrm{O}_{4}(47 \% \mathrm{wt})$ and $(\mathrm{Cu}, \mathrm{Fe}) \mathrm{O}(20 \% \mathrm{wt})$. The NPs were dispersed in ethanol on a lacey carbon TEM grid. The analyses were performed with an objective lens aberration corrected FEI Titan TEM operated at $300 \mathrm{kV}$ and equipped with an annular detector and a Gatan Quantum image filter.

Fig. 1a shows nanoparticles decorated with a secondary phase. X-ray diffraction (not shown) combined with the HREM confirms that the NPs adopt the cubic and tetragonal phase of $\mathrm{Cu}$ ferrites (Fig. 1b). However, the low crystallinity of the secondary phase (Fig. 1c) makes it difficult to identify it using HREM. Wet tried using the $\mathrm{Cu} \mathrm{L}_{2,3}-\mathrm{Fe} \mathrm{L}_{2,3}$ signal to generate a bivariate histogram to isolate the $\mathrm{Cu}$-rich regions of the SI, but a good compromise between the signal-to-noise ratio (SNR) and the "purity" of the metallic $\mathrm{Cu}$ character could not be achieved. Given the scarcity of pure $\mathrm{Cu}$ pixels, various versions of Independent Component Analysis [1] failed to produce physically sound components although the Bayesian Linear Unmixing with a NFINDR initialization [2] showed potential.

We used a Multivariate Curve Resolution (MCR) algorithm to extract physically meaningful spectra $(S)$ and composition maps $(C)$ from an image $(X)$. The loglikelihood variant (MCR-LLM) has been shown to perform particularly well in low SNR datasets [3]. In this work, a hierarchical version of MCR-LLM was used to deal with local composition gradients and faulty pixels. Fig. 1d illustrates the methodology in which spectral features are extracted dyadically with each step. The $137 \times 93$ spectra were thus reduced to 331 significant ones from which we selected 4 (Fig. 1e): $\mathrm{Cu}$ (red), $\mathrm{Cu}$ ferrites (green), carbon (blue: featureless exponential background) and the hole (yellow: noise). The maps (Fig. 1f) associated with each spectrum were generated by finding the set coefficients that maximizes the loglikelihood [3]. This method is especially suited for datasets with low signal-to-noise levels contaminated with Poisson noise.

The EELS Fe:Cu:O intensity ratio of the $\mathrm{Cu}$ ferrite phase is consistent with $\mathrm{Cu}_{x} \mathrm{Fe}_{3-\mathrm{x}} \mathrm{O}_{4}(0 \leq x \leq 1)$. The $\mathrm{Cu}$ content of the metallic $\mathrm{Cu}$ approached $70 \%$, but was found along $\mathrm{Fe}(10 \%$ at) and $\mathrm{O}(20 \%$ at). The $\mathrm{Cu}$ $\mathrm{L}_{2,3}$ edge profile is mostly metallic and the shape of the $\mathrm{O}$ Kedge is indicative of the presence of $(\mathrm{Cu}, \mathrm{Fe}) \mathrm{O}$, demonstrating that metallic $\mathrm{Cu}$ is mixed with the monoxyde. Comparing the spatial distribution of $\mathrm{Cu}$ clusters (Fig. 1f) with the Sobel-filtered STEM image taken from the same ROI (Fig. 1g), it can be inferred that the protrusions on the $\mathrm{Cu}$ ferrites NPs correspond to $\mathrm{Cu}$-rich zones. The presence of $\mathrm{Cu}$ buds on the $\mathrm{Cu}$ ferrites are sought to form during solidification of the liquid droplets as the excess $\mathrm{Cu}$ is expelled. $\mathrm{Cu}$ then oxidizes in presence of the residual oxygen in the reactor or the air.

Unlike traditional methods, the exponential background is conserved and there is no need to sacrifice 
spatial resolution to increase the signal-to-noise ratio. In this case, a precise evaluation of the composition is achieved by the analysis of the endmember spectra rather than the background-subtracted signal of individual pixel. This work highlights the multiple opportunities of deep data analysis provided by advanced latent variable decomposition methods for the optimal interpretation of SIs [4].

References:

[1] N. Bonnet and D. Nuzillard. Ultramicroscopy 102 (2005), p. 327

[2] N. Dobigeon and N. Brun. Ultramicroscopy 120 (2012), p. 25.

[3] F. Lavoie, N. Braidy and R. Gosselin Chemotr Intell Lab 153 (2016), p. 40

[4] The authors acknowledge funding from the NSERC, FRQ and the Canada Research Chair programs. We thank Andreas Korinek from the Canadian Center for Electron Microscopy for the data acquisition.
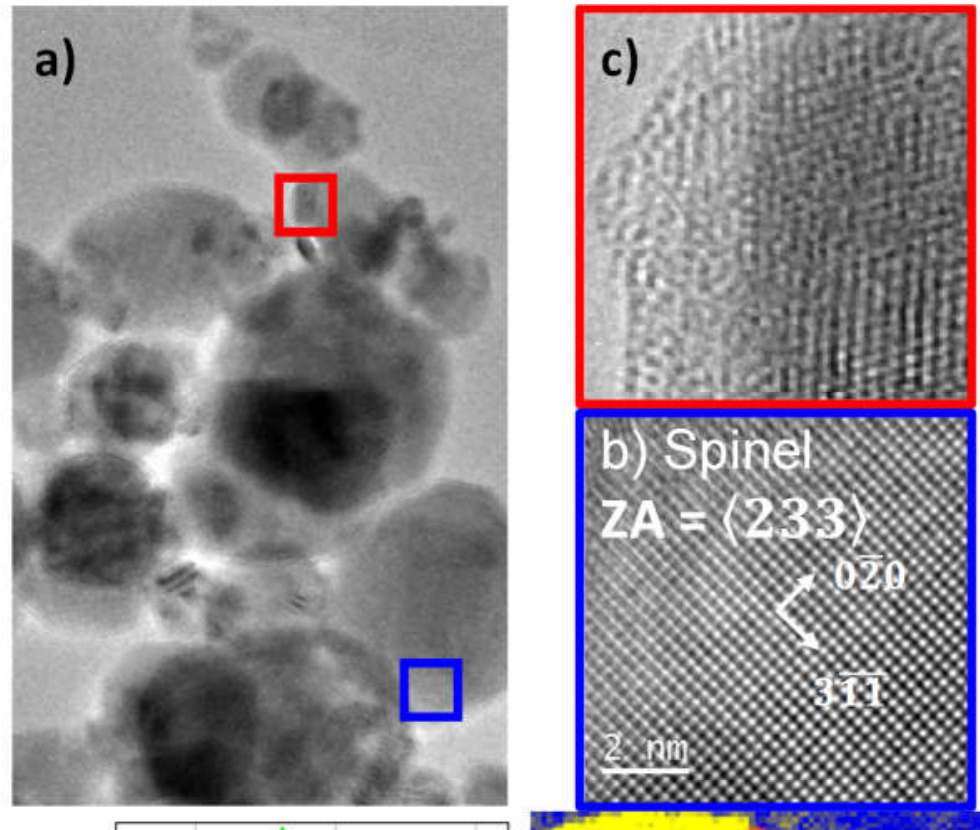

d)
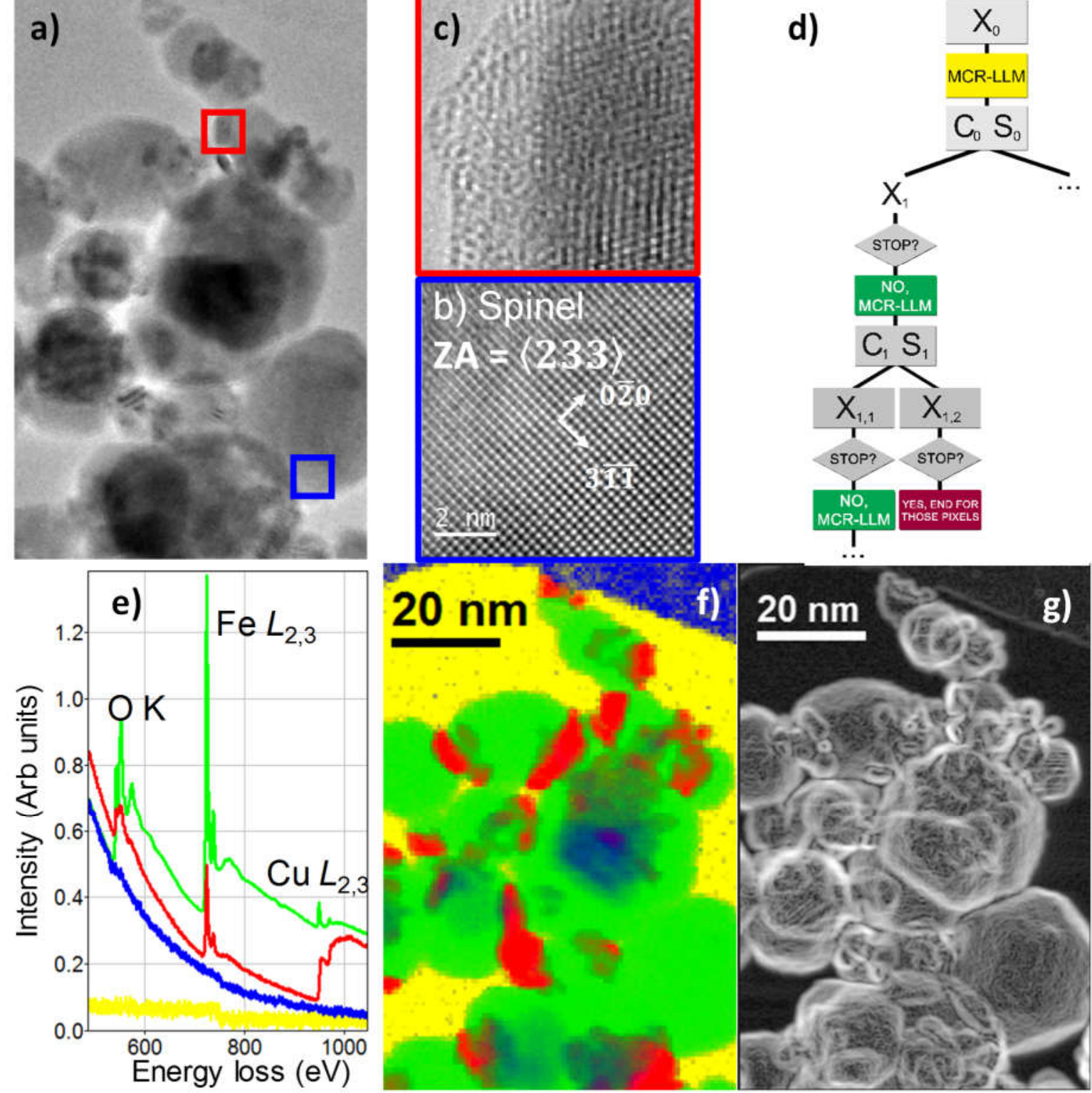

Figure 1. (a) HREM micrograph. HREM image of a Cu ferrite nanoparticle (b) and a Cu-rich zone (c). (d) Algorithm used for the hierarchical method for classifying the spectra. (e)EELS spectra of the 4 characteristic regions (Green: Cu ferrite, Red: $\mathrm{Cu}, \mathrm{CuO}$, Blue: carbon lacey film and Yellow: hole). (g) Sobel-filtered STEM HAADF image of the same ROI as (a) and (f). 